\title{
The Monte Carlo Simulation of Secondary Electrons Excitation in the Resist PMMA
}

\author{
Xiao Pei ${ }^{1}$ \\ ${ }^{1}$ Jiangsu University of Science and Technology, Zhangjiagang campus, Zhangjiagang, Jiangsu, China \\ Correspondence: Xiao Pei, Jiangsu University of Science and Technology, Zhangjiagang campus, Zhangjiagang \\ 215600, Jiangsu, China. E-mail: pxiao@ustc.edu
}

Received: February 21, 2014 Accepted: March 17, 2014 Online Published: March 31, 2014

doi:10.5539/apr.v6n3p1 URL: http://dx.doi.org/10.5539/apr.v6n3p1

\begin{abstract}
The Monte Carlo method was used to simulate the process of secondary electrons excitation in resist PMMA with Mott cross section and dielectric function model. By analyzing the characteristics of secondary electrons excitation in the resist PMMA, and the simulation of secondary electrons energy range, we hold the opinion that the secondary electrons can not be ignored in the electron beam lithography.
\end{abstract}

Keywords: secondary electrons, electron beam lithography (EBL), Monte Carlo simulation, dielectric function

\section{Introduction}

Electron-beam lithography (EBL) is widely used to achieve high resolution patterns in nanotechnology research and development. Because it can break the diffraction limit of conventional optical lithography, with the characteristics of high-resolution EBL has broad application prospects, such as to produce the mask and three dimensional photonic crystals. EBL can be divided into high-energy EBL and low-energy EBL $(0.5 \mathrm{keV}-5$ $\mathrm{keV}$ ). Since the limits of heat and proximity effect (Nilsson, 2011; Manfrinato et al., 2013) restricted the development of high-energy EBL, so the low- energy EBL more potential (Beaumont, Dubuc, Beauvais, Drouin, \& Drouin, 2010; Yang et al., 2006). EBL is to focus the accelerated electrons incident on the substrate which coated with resist material such as Polymethyl methacrylate (PMMA). The chemical reactions will be occurred in PMMA because the electronic energy deposition. The image can be formed by chemical solvents action. Computer simulations for EBL (Rommel et al., 2012; Sun, You, Xiao, \& Ding, 2006; Zhao, S. Y. Lee, S. H. Lee, Kim, \& Cho, 2012), is to simulate the electron energy loss distribution in PMMA. According to the electron energy loss distribution can infer the change in resist. In conventional EBL simulation, usually not included secondary electron excitation. Because the high-energy electron beam incident on material, the secondary electron excitation efficiency is low, no effect on the simulation results. But the low-energy electron beam incident, the impact of secondary electrons is more significant. Therefore, this paper used the most successful model, dielectric function, to simulate the secondary electron excitation in PMMA and to analyze the characteristics of secondary electron excitation.

\section{Model}

Monte Carlo method to determine the trajectories and energy loss of each electron which incident on material use random number. Through the large number of electronic statistics, the high accuracy of the results obtained. When electrons incident to the solid will be collided with atoms, we called scattering. Scattering is divided into elastic scattering and inelastic scattering. Elastic scattering is the collision of electrons and atom nuclei, only to change the direction of electrons, does not change the electron energy. Inelastic scattering is the collision of electrons and extranuclear electron, both change the direction and electron energy. Energy loss of inelastic is divided into two parts, part of the energy loss absorbed by material, the other excite secondary electrons.

\subsection{Elastic Scattering}

In order to accurately simulate the elastic scattering process the Mott scattering cross section is used. Studies ( $\mathrm{Pu}$, Ding, Sun, \& Wu, 2004) have shown that Mott scattering cross section have an insurmountable advantages compared other methods, especially in the low-energy collisions of electrons and low atomic number material. Mott cross section contains the electron spin. 


$$
\frac{d \sigma}{d \Omega}=|f(\theta)|^{2}+|g(\theta)|^{2}
$$

$f(\theta)$ and $g(\theta)$ are the scattering amplitudes

$$
\begin{gathered}
f(\theta)=\frac{1}{2 i K} \sum_{l=0}^{\infty}\left\{(l+1)\left(e^{i 2 \delta \delta_{t}^{+}}-1\right)+l\left(e^{i 2 \delta_{l}^{-}}-1\right)\right\} P_{l}(\cos \theta) \\
g(\theta)=\frac{1}{2 i K} \sum_{l=1}^{\infty}\left\{-e^{i 2 \delta_{l}^{+}}+e^{i 2 \delta_{l}^{-}}\right\} P_{l}^{1}(\cos \theta)
\end{gathered}
$$

$\hbar K$ is scattering electron momentum, $K=\sqrt{E^{2}-m^{2} c^{4}} / \hbar c$, where $\delta_{l}^{+}$and $\delta_{l}^{-}$are spin "up" and spin "down" phase shifts of 1-th partial wave respectively, which can be calculated by using partial wave expansion method. Where $P_{l}(\cos \theta)$ and $P_{l}^{1}(\cos \theta)$ are Legendre and first order associated Legendre function, respectively.
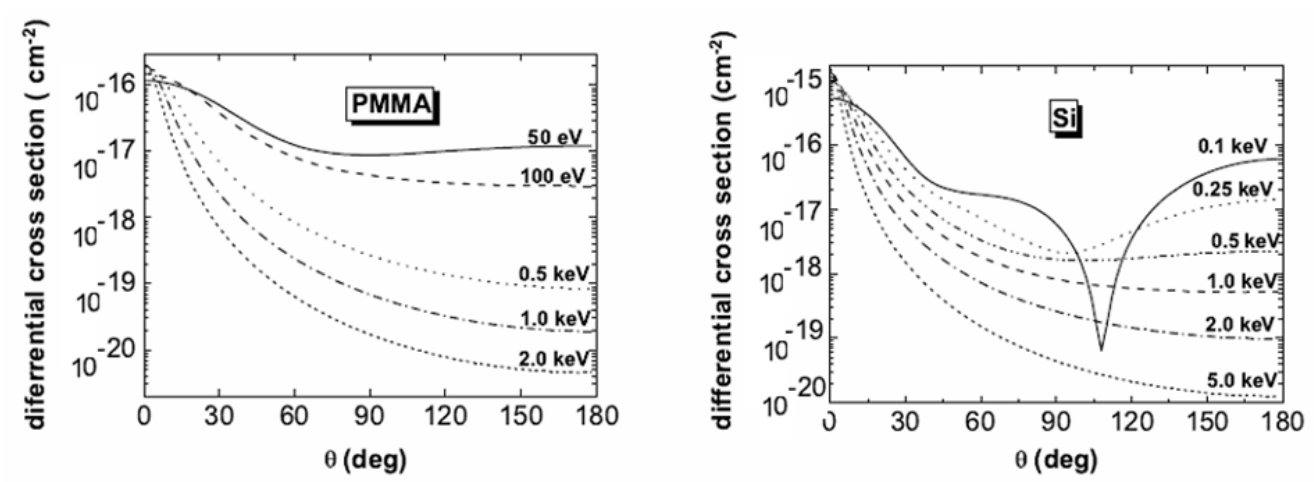

Figure 1. Mott differential scattering cross section of PMMA and Si

In Figure 1, at low energies differential scattering cross section appears fine structure, the performance of electron emission angle in a certain priority oriented by elastic scattering. Fine structures of PMMA began to appear in the $50 \mathrm{eV}$, and Si appears in the $250 \mathrm{eV}$. For low-energy electron beam lithography the fine structure of differential cross section influence the energy distribution in different directions, therefore can not be ignored, particularly when the substrate is a high atomic number material.

\subsection{Inelastic Scattering}

Based on the Continuous Slowing Down Approximation (CSDA) (Sun et al., 2002) method is used to describe inelastic scattering formerly. CSDA method is not well suited to simulate low-energy electron scattering processes, because it is no longer applicable when energy was below the average atomic ionization energy. In addition, this method can not simulate a cascade secondary electrons produced. However, Penn's dielectric function method for inelastic scattering and secondary electron excitation match well the experimental results (Ding \& Shimizu, 1996; Zhang, Wang, Li, Mao, \& Ding, 2012). Therefore, this method is considered to be the most successful simulated secondary electron excitation model (Ding \& Shimizu, 1996). The differential cross sections of inelastic scattering based on optical energy loss function is expressed as:

$$
\frac{d \lambda^{-1}}{d(\Delta E)}=\frac{1}{2 \pi a_{0} E \Delta E} \int_{0}^{\infty} \frac{\hbar \omega_{p}}{\Delta E-\hbar \omega_{p}} \operatorname{Im}\left\{\frac{-1}{\varepsilon\left(\omega_{p}\right)}\right\} d\left(\hbar \omega_{p}\right) \Theta\left[\frac{\hbar}{2 m}\left(2 k \bar{q}-\bar{q}^{2}\right)-\Delta E\right]
$$

$\lambda$ is Inelastic scattering mean free path, $E$ is electron energy, $\Delta E$ is the energy loss, $\operatorname{Im}\{-1 / \varepsilon(\omega)\}$ optical energy loss function, $\bar{q}=\sqrt{2 m /\left(\Delta E-\hbar \omega_{p}\right)}$. This scattering cross-section is defined as the probability that electron loses energy for unit path length traveled in solids. It is noted that the optical energy loss function of the material determines the inelastic scattering cross section. The optical energy loss function of PMMA can be obtained from the experimental data measured directly in lower energy region. However, high-energy radiation damage occurs PMMA decomposition, the results of experimental measurements are no longer credible. The data used in the paper is less than $33 \mathrm{eV}$ taken from experimental data (Ritsko, Brillson, Bigelow, \& Fabish, 1978), when more than $33 \mathrm{eV}$, calculated from the optical absorption data of each element in proportion. Figure 2 shows the optical energy loss function of PMMA. 


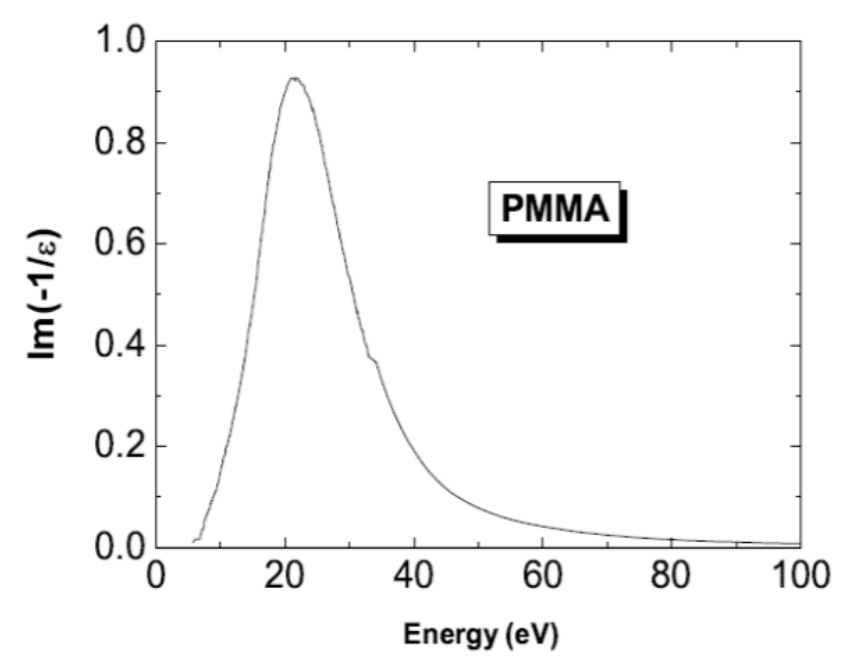

Figure 2. The optical energy loss function of PMMA

\subsection{Monte Carlo Method}

The individual electron scattering progresses is schematically shown in Figure 3. In our approach the scattering angle, energy loss, step length and so forth are determined by random number $R \in[0,1]$ in a particular scattering event. The mean free path describe the average length between two successive collision events. The total mean free path can be expressed as

$$
\lambda_{t}^{-1}=\lambda_{e l}^{-1}+\lambda_{\text {in }}^{-1}
$$

So the step length, $\mathrm{s}$, obeys to Possion stochastic, can be get by a random number $R_{1}$

$$
\left\{\begin{array}{c}
f(s)=\lambda_{t}^{-1} e^{-s / \lambda_{t}} \\
s=-\lambda_{t} \ln R_{1}
\end{array}\right.
$$

Another random, $R_{2}$, be selected to determine which type scattering, if

$$
R_{2}<\lambda_{e l}^{-1} / \lambda_{t}^{-1}
$$

Elastic scattering, inelastic scattering otherwise.

For elastic scattering, when the specimen is compound another random number $R_{3}$ will be used to determine which element be attributed to.

$$
\frac{\sum_{j=1}^{i-1} C_{j}^{a} / \lambda_{e l}^{j}}{1 / \lambda_{e l}}<R_{3}<\frac{\sum_{j=1}^{i} C_{j}^{a} / \lambda_{e l}^{j}}{1 / \lambda_{e l}}
$$

The angle of elastic scattering is determined by the equation

$$
R_{4}=\int_{0}^{\theta} \frac{d \sigma}{d \Omega} \sin \theta^{\prime} d \theta^{\prime} / \int_{0}^{\pi} \frac{d \sigma}{d \Omega} \sin \theta^{\prime} d \theta^{\prime}
$$

For the inelastic scattering, we determine the amount of energy loss $\Delta E$ by

$$
R_{5}=\int_{0}^{\Delta E} \frac{d \lambda_{i n}^{-1}}{d\left(\Delta E^{\prime}\right)} d\left(\Delta E^{\prime}\right) / \int_{0}^{E-E_{F}} \frac{d \lambda_{i n}^{-1}}{d\left(\Delta E^{\prime}\right)} d\left(\Delta E^{\prime}\right)
$$

Once get the energy loss $\Delta E$ the inelastic scattering angle is determined by

$$
R_{6}=\int_{0}^{\theta} \frac{d^{2} \lambda_{i n}^{-1}}{d \Omega d(\Delta E)} \sin \theta^{\prime} d \theta^{\prime} / \int_{0}^{\pi} \frac{d^{2} \lambda_{i n}^{-1}}{d \Omega d(\Delta E)} \sin \theta^{\prime} d \theta^{\prime}
$$

and the azimuthal angle is assumed to be

$$
\phi=2 \pi R_{7}
$$




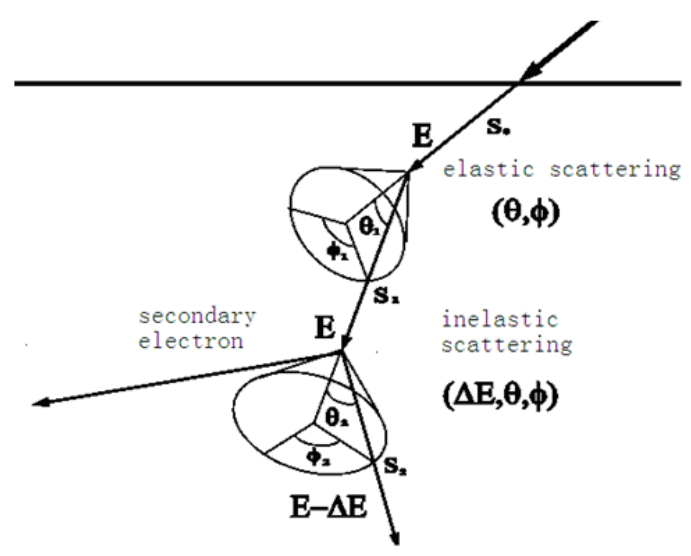

Figure 3. Schematic diagram of electron scattering

\section{Results and Discussion}

When electron penetrates into solid it will undergo hundreds or thousands of scattering events. Each electron trajectory is followed until its kinetic energy falls below the threshold energy or electron flight out of the surface. The individual electron scattering progresses is schematically shown in Figure 1. In our approach the scattering angle, energy loss, step length and so forth are determined by random number in a particular scattering event. Every inelastic collisions will lose energy $\Delta E$, this moment producing a secondary electron. Its angle is determined by random number and the principle of momentum conservation and the energy $E=\Delta E-E_{B}$ ( $E_{B}$ is the shell binding energy). Once secondary electrons are excited, its energy, the direction of movement and the coordinates is stored. When the simulation of incident electrons is finished, the information of secondary electrons is recalled and is simulated same as the incident electron. A large number of lower energy cascade secondary electrons are generated. Simulations will be carried out until the secondary electron escape from the surface of sample or be absorbed. The number of secondary electrons significantly greater than incident electrons, This is because each time inelastic scattering a secondary electron produced, movement of the secondary electron cascade will produce more secondary electrons, much larger than the number of incident electrons.

Figure 4 shows the depth distribution of the secondary electrons produced. Values in the Figure 4 are normalized. Secondary electron is accompanied electron energy loss, so the probability of the maximum depth of the secondary electron generation is also the maximum depth of electron energy loss probability. The greater energy of incident electron, the greater depth of maximum probability of electron energy loss and the greater depth of secondary electron distribution. Whatever energy of incident electron the secondary electrons are generated in the low energy region. When energy is larger than $50 \mathrm{eV}$, the amount of secondary electrons generated is small. Electron energy is smaller, the greater the inelastic scattering cross section. Inelastic scattering probability of occurrence increases. The generation of secondary electron is accompanied electrons energy loss, so the excitation of secondary electrons in the low energy region more concentrated. The distribution of secondary electrons was simulated in Figure 5. It is clear that low-energy electrons incident on, secondary electrons are mainly concentrated in the depth of several tens of nanometers to hundreds of nanometers, which is the optimum thickness of the PMMA material. 


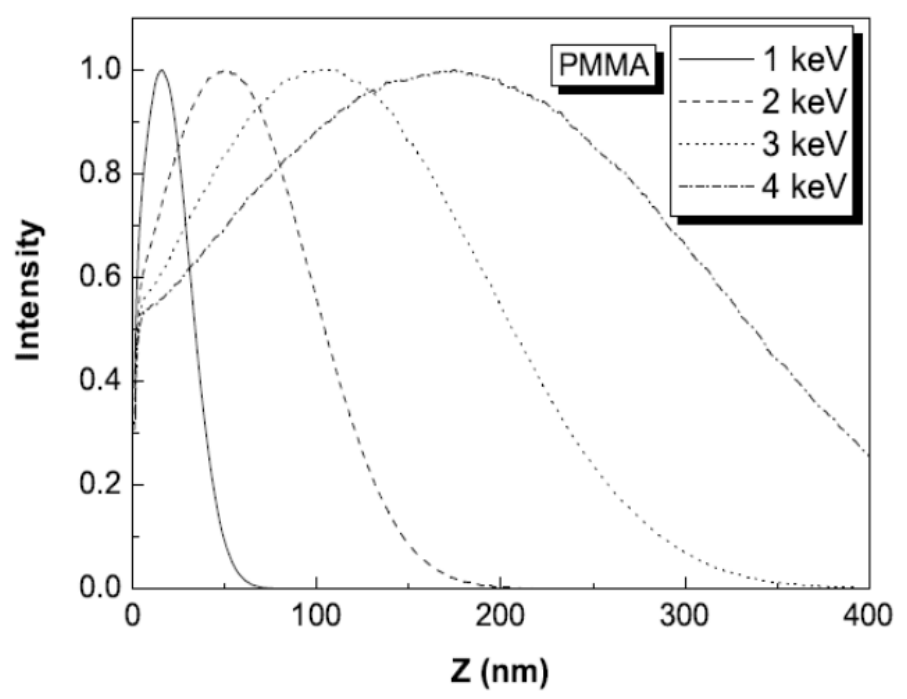

Figure 4 . The depth distribution of secondary electrons

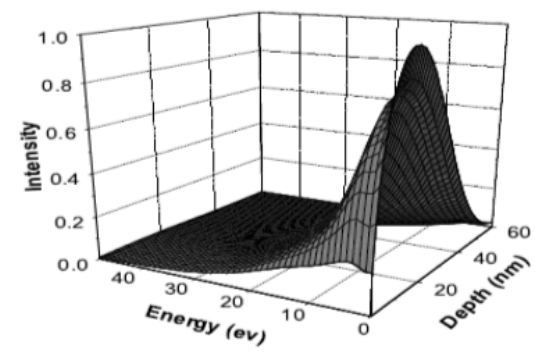

(a) $1 \mathrm{keV}$

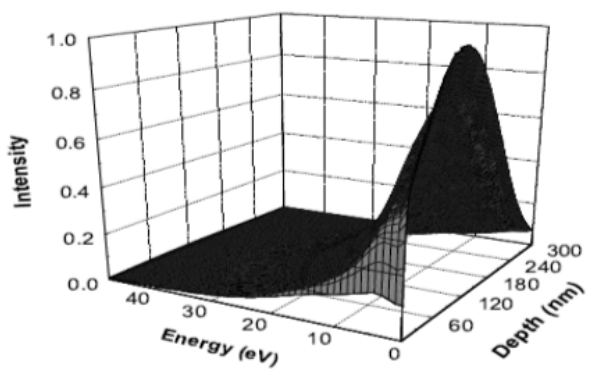

(c) $3 \mathrm{keV}$



(b) $2 \mathrm{keV}$



(d) $4 \mathrm{keV}$

Figure 5. The distribution of secondary electrons in depth and energy

Although the energy of secondary electron is low, but the energy of a few electron volts can react chemically of PMMA material and the number of secondary electron is huge, so there will be some impact on the energy loss distribution. In Figure 6, with substrate Si, $100 \mathrm{~nm}$ PMMA and $3 \mathrm{keV}$ incident electron, the electron energy loss distribution is obviously different. Include the secondary electron excitation, will make the computer run time increased several times. But if accurate simulations of the electron beam etching, it should include secondary electron excitation. This will make the simulation results more credible. 




Figure 6. The energy loss distribution, line: no include secondary electrons; dot: include secondary electrons

\section{Conclusions}

In this paper, the Mott scattering cross section and the dielectric function model are used to simulate the process of scattering and secondary electron excitation in the material. We studied the properties of the secondary electrons, and calculated the energy and depth distribution of secondary electrons excitation. We found that the secondary electrons excitation accompanied the electron energy loss and inelastic scattering. Whatever energy of incident electron the secondary electrons are generated in the low energy region. Large number of cascade secondary electrons energy was low, but the energy is sufficient to react the PMMA material. If improving the accuracy of the simulation results, the secondary electrons are generated should be taken into account.

\section{References}

Beaumont, A., Dubuc, C., Beauvais, J., \& Drouin, D. (2010). Direct-write electron beam lithography in silicon dioxide at low energy. Journal of Vacuum Science \& Technology B, 28(5), 940-945. http://dx.doi.org/10.1116/1.3478304

Ding, Z. J., \& Shimizu, R. (1996). A Monte Carlo modeling of electron interaction with solids including cascade secondary electron production. Scanning, 18(2), 92-113. http://dx.doi.org/10.1002/sca.1996.4950180204

Manfrinato, V. R., Zhang, L. H., Su, D., Duan, H. G., Hobbs, R. G., Stach, E. A., \& Berggren, K. K. (2013). Resolution Limits of Electron-Beam Lithography toward the Atomic Scale. Nano Letters, 13(4), 1555-1558. $\mathrm{http}: / / \mathrm{dx}$.doi.org/10.1021/nl304715p

Nilsson, B. A. (2011). Experimental evaluation method of point spread functions used for proximity effects correction in electron beam lithography. Journal of Vacuum Science \& Technology B, 29(6), 06F311. http://dx.doi.org/10.1116/1.3656343

Pu, Q. R., Ding, Z. J., Sun, X., \& Wu, Z. Q. (2004). Use of Mott cross section in the simulation of electron scattering and transmission through mask in a projection lithography system. Journal of Chinese Electron Microscopy Society, 23(5), 571-574. http://dx.doi.org/10.3969/j.issn.1000-6281.2004.05.012

Ritsko, J. J., Brillson, L. J., Bigelow, R. W., \& Fabish, T. J. (1978). Electron energy loss spectroscopy and the optical properties of PMMA from 1 to $300 \mathrm{eV}$. The Journal of Chemical Physics, 69(9), 3931-3939. http://dx.doi.org/10.1063/1.437131

Rommel, M., Hoffmann, K. E., Reindl, T., Weis, J., Unal, N., \& Hofmann, U. (2012). Benchmark test of Monte-Carlo simulation for high resolution electron beam lithography. Microelectronic Engineering, 98, 202-205. http://dx.doi.org/10.1016/j.mee.2012.07.032

Sun, X., Ding, Z. J., Pu, Q. R., Li, H. M., Wu, Z. Q., Gu, W. Q., ... Kang, N. K. (2002). Monte Carlo simulation of electron transmission through the scattering masks with angular limitation for projection electron lithography. Journal of Applied Physics, 92(7), 3641-3646. http://dx.doi.org/10.1063/1.1505679

Sun, X., You, S. F., Xiao, P., \& Ding, Z. J. (2006). Simulation of the proximity effect of electron beam lithography. Acta Physica Sinica, 55(1), 148-154. Retrieved from http://wulixb.iphy.ac.cn/CN/Y2006/V55/I1/148

Yang, H., Jin, A., Luo, Q., Gu, C., Cui, Z., \& Chen, Y. (2006). Low-energy electron-beam lithography of 
hydrogen $\quad$ silsesquioxane. $\quad$ Microelectronic $\quad$ Engineering, $\quad 83(4-9), \quad$ 788-791. http://dx.doi.org/10.1016/j.mee.2006.01.004

Zhang, P., Wang, H. Y., Li, Y. G., Mao, S. F., \& Ding, Z. J. (2012). Monte Carlo Simulation of Secondary Electron Images for Real Sample Structures in Scanning Electron. Microscopy. Scanning, 34(3), 145-150. http://dx.doi.org/10.1002/sca.20288

Zhao, X. Y., Lee, S. Y., Lee, S. H., Kim, B. G., \& Cho, H. K. (2012). Fast simulation of stochastic exposure distribution in electron-beam lithography. Journal of Vacuum Science \& Technology B, 30(6), 06F308. http://dx.doi.org/10.1116/1.4767447

\section{Copyrights}

Copyright for this article is retained by the author(s), with first publication rights granted to the journal.

This is an open-access article distributed under the terms and conditions of the Creative Commons Attribution license (http://creativecommons.org/licenses/by/3.0/). 